astro-ph/0110225

CERN-TH/2001-264

MIT-CTP-3181, UCI-TR-2001-26

UMN-TH-2026/01, TPI-MINN-01/44

\title{
Prospects for Detecting Supersymmetric Dark Matter at Post-LEP Benchmark Points
}

\author{
John Ellis ${ }^{1}$, Jonathan L. Feng ${ }^{2,3}$, Andrew Ferstl ${ }^{4}$, \\ Konstantin T. Matchev ${ }^{1}$ and Keith A. Olive ${ }^{5}$ \\ ${ }^{1}$ TH Division, CERN, CH-1211 Geneva 23, Switzerland \\ ${ }^{2}$ Center for Theoretical Physics, \\ Massachusetts Institute of Technology, Cambridge, MA 02139, USA \\ ${ }^{3}$ Department of Physics and Astronomy, \\ University of California, Irvine, CA 92697, USA \\ ${ }^{4}$ Department of Physics, Winona State University, Winona, MN 55987, USA \\ ${ }^{5}$ Theoretical Physics Institute, School of Physics and Astronomy, \\ University of Minnesota, Minneapolis, MN 55455, USA
}

\begin{abstract}
A new set of supersymmetric benchmark scenarios has recently been proposed in the context of the constrained MSSM (CMSSM) with universal soft supersymmetry-breaking masses, taking into account the constraints from LEP, $b \rightarrow s \gamma$ and $g_{\mu}-2$. These points have previously been used to discuss the physics reaches of different accelerators. In this paper, we discuss the prospects for discovering supersymmetric dark matter in these scenarios. We consider direct detection through spin-independent and spin-dependent nuclear scattering, as well as indirect detection through relic annihilations to neutrinos, photons, and positrons. We find that several of the benchmark scenarios offer good prospects for direct detection via spin-independent nuclear scattering and indirect detection via muons produced by neutrinos from relic annihilations inside the Sun, and some models offer good prospects for detecting photons from relic annihilations in the galactic centre.
\end{abstract}

October 2001 


\section{Introduction}

After the closure of LEP, at the start of Run II of the Tevatron Collider, with the LHC experimental programme being prepared, and linear $e^{+} e^{-}$collider projects under active discussion, now is an appropriate time to review the available experimental constraints on supersymmetry and assess the prospects for its discovery. In parallel with present and future accelerator projects, many non-accelerator experiments that may contribute to the search for supersymmetry are underway or in preparation. These include direct searches for the elastic scattering of astrophysical cold dark matter particles on target nuclei, and indirect searches for particles produced by the annihilations of supersymmetric relic particles inside the Sun or Earth, in the galactic centre or in the galactic halo.

A set of benchmark supersymmetric model parameter choices was recently proposed [1] with the idea of exploring the possible phenomenological signatures in different classes of experiments in a systematic way. The proposed benchmark points were chosen by first implementing the constraints on the CMSSM parameter space [2] imposed by previous experiments, such as the searches for sparticles [3] and Higgs bosons at LEP [4] and elsewhere, the measured rate for $b \rightarrow s \gamma$ decay [5], and (optionally) the value of $g_{\mu}-2$ recently reported by the BNL E821 experiment [6]. The CMSSM parameter space was also constrained by requiring the calculated supersymmetric relic density to fall within the range $0.1<\Omega_{\chi} h^{2}<0.3$ preferred by astrophysics and cosmology. Four general regions of allowed parameter space were identified: a 'bulk' region at relatively low $m_{0}$ and $m_{1 / 2}$, a 'focus point' region $[7,8]$ at relatively large $m_{0}$, a coannihilation 'tail' extending out to relatively large $m_{1 / 2}[9,10]$, and a possible 'funnel' between the focus point and coannihilation regions due to rapid annihilation via direct-channel Higgs boson poles [11].

The benchmark points were chosen not to provide an unbiased statistical sample of the CMSSM parameter space, which is in any case difficult to define in the absence of any unbiased a priori measure, but rather to select representative examples of different possibilities that cannot yet be logically excluded. Note that while these scenarios are confined to the context of supergravity, they span a large range of dark matter properties. While other supersymmetry-breaking schemes lead to a variety of collider signals, with respect to dark matter, they often predict vanishing or highly suppressed thermal relic densities for the most natural candidate, the neutralino. These alternative scenarios therefore typically have no viable dark matter candidates, at least without additional structure and an accompanying loss of predictability.

Of the 13 benchmark points, B, C, G, I, and L lie within the 'bulk' region; E and F are 

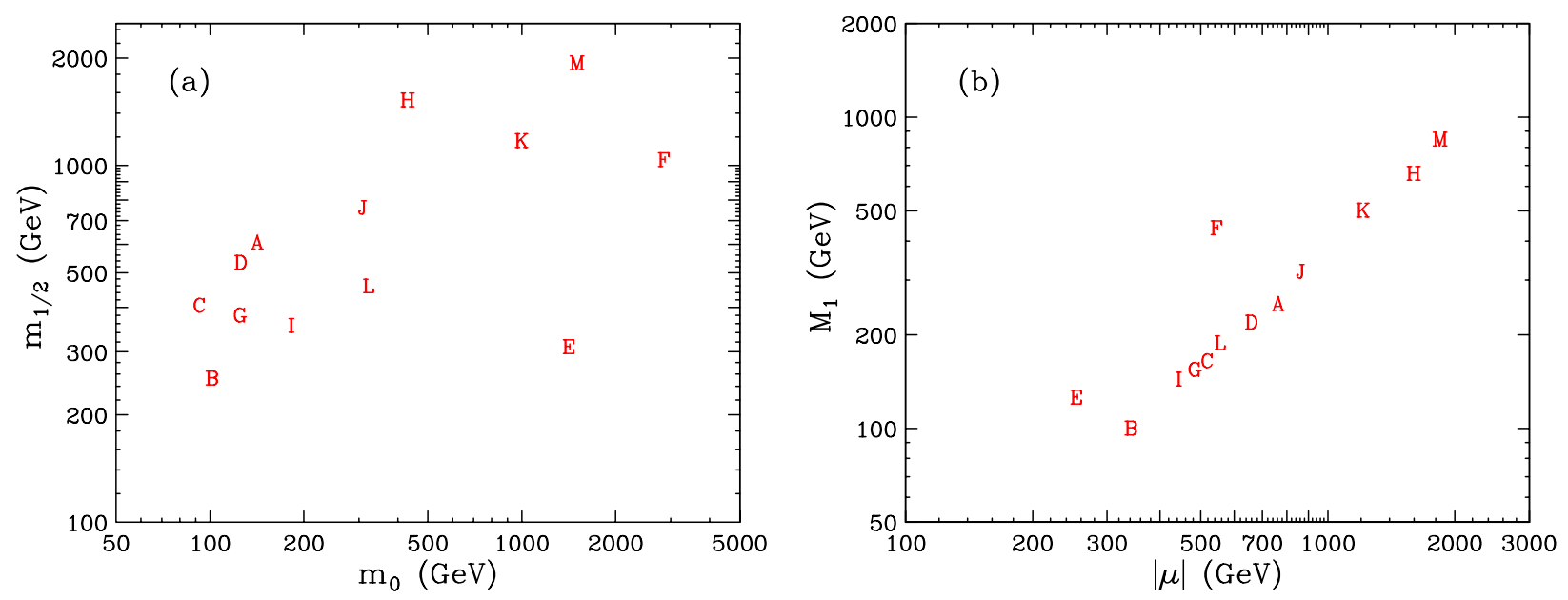

Figure 1: Benchmark points [1] in the (a) $\left(m_{0}, m_{1 / 2}\right)$ and (b) $\left(|\mu|, M_{1}\right)$ planes.

in the focus point region; A, D, H, and J are strung out along the coannihilation tail; and $\mathrm{K}$ and $\mathrm{M}$ are chosen at (relatively) large $m_{1 / 2}$ and $m_{0}$, in the rapid annihilation funnel regions. About half of the proposed points yield a value of $g_{\mu}-2$ within two standard deviations of the value reported by BNL E821, but we did not impose this as an absolute requirement. For example, two points with $\mu<0$, the sign disfavoured by $g_{\mu}-2$, were retained. Fig. 1 provides an overview of the locations of the benchmark points in the $\left(m_{0}, m_{1 / 2}\right)$ and $\left(|\mu|, M_{1}\right)$ planes. We see that the proposed scenarios mainly have $m_{1 / 2}>m_{0}$, except for the two focus point models $\mathrm{E}$ and $\mathrm{F}$. These also have larger values of $M_{1} /|\mu|$, and therefore more Higgsino-like lightest supersymmetric particles (LSPs). Table 1 displays many properties of the proposed scenarios, including the LSP mass, its gaugino composition, its cosmological relic density, and rates for the many astrophysical signatures to be discussed in subsequent sections of this paper.

It was found previously [1] that, in the $g_{\mu}-2$-friendly scenarios, supersymmetry was relatively easy to discover and study at future colliders such as the LHC and a linear collider with $E_{C M}=1 \mathrm{TeV}$, which would be able to observe rather complementary subsets of CMSSM particles. However, some of the other points might escape detection, except via observations of the lightest neutral Higgs boson of the CMSSM. The most difficult points were typically those in the focus point region, at the tip of the coannihilation tail, or along the rapidannihilation funnels, with points $\mathrm{F}, \mathrm{H}$, and $\mathrm{M}$ being particularly elusive.

In this paper, we report on the prospects for the direct and indirect detection of astrophysical dark matter for each of these benchmark points. We present cross sections for the elastic scattering of supersymmetric relic particles off both protons and neutrons via 


\begin{tabular}{|c|c|c|c|c|c|c|c|c|c|c|c|c|c|}
\hline Model & A & B & $\mathrm{C}$ & $\mathrm{D}$ & $\mathrm{E}$ & $\mathrm{F}$ & G & $\mathrm{H}$ & I & $\mathrm{J}$ & K & $\mathrm{L}$ & M \\
\hline$m_{1 / 2}$ & 613 & 255 & 408 & 538 & 312 & 1043 & 383 & 1537 & 358 & 767 & 1181 & 462 & 1953 \\
\hline$m_{0}$ & 143 & 102 & 93 & 126 & 1425 & 2877 & 125 & 430 & 188 & 315 & 1000 & 326 & 1500 \\
\hline $\tan \beta$ & 5 & 10 & 10 & 10 & 10 & 10 & 20 & 20 & 35 & 35 & 39.6 & 45 & 45.6 \\
\hline $\operatorname{sgn}(\mu)$ & + & + & + & - & + & + & + & + & + & + & - & + & + \\
\hline$m_{\chi}$ & 251.8 & 98.1 & 163.8 & 221.0 & 119.2 & 434.2 & 153.7 & 663.6 & 143.1 & 320.8 & 505.7 & 188.0 & 853.9 \\
\hline$R_{\chi}$ & 0.997 & 0.986 & 0.994 & 0.997 & 0.954 & 0.950 & 0.994 & 0.999 & 0.993 & 0.998 & 0.999 & 0.995 & 0.999 \\
\hline$\Omega h^{2}$ & 0.26 & 0.18 & 0.14 & 0.19 & 0.31 & 0.17 & 0.16 & 0.29 & 0.16 & 0.20 & 0.19 & 0.21 & 0.17 \\
\hline$\sigma_{P}^{\mathrm{sc}}$ & 387.9 & 6567. & 1031. & 1.745 & 4859. & 4121. & 2262 . & 32.11 & 8953. & 335.3 & 0.061 & 5862. & 32.61 \\
\hline$\sigma_{P}^{\mathrm{sp}}$ & 0.260 & 11.06 & 1.622 & 0.518 & 102.4 & 14.15 & 2.236 & 0.022 & 3.045 & 0.216 & 0.075 & 1.358 & 0.016 \\
\hline$\sigma_{N}^{\mathrm{sc}}$ & 399.8 & 7002 . & 1085. & 2.304 & 5004. & 4221. & 2426. & 33.19 & 9730. & 357.5 & 0.192 & 6375. & 34.04 \\
\hline$\sigma_{N}^{\mathrm{sp}}$ & 0.224 & 8.750 & 1.331 & 0.434 & 64.19 & 8.831 & 1.805 & 0.017 & 2.416 & 0.171 & 0.055 & 1.053 & 0.012 \\
\hline$\Phi_{\mu}^{\odot}$ & 0.0138 & 5.43 & 0.706 & 0.0585 & 152. & 7.25 & 1.23 & $10^{-5}$ & 1.809 & 0.0493 & 0.0089 & 1.002 & 0.0013 \\
\hline$\Phi_{\mu}^{\oplus}$ & $10^{-9}$ & $10^{-5}$ & $10^{-7}$ & $10^{-13}$ & $10^{-5}$ & $10^{-5}$ & $10^{-6}$ & $10^{-12}$ & $10^{-4}$ & $10^{-8}$ & $10^{-13}$ & $10^{-4}$ & $10^{-10}$ \\
\hline$\Phi_{\gamma}^{1}$ & 1.428 & 84.29 & 10.19 & 2.248 & 85.59 & 39.60 & 63.90 & 0.204 & 535.0 & 25.86 & 119.4 & 992.4 & 37.48 \\
\hline$\Phi_{\gamma}^{50}$ & 0.340 & 0.874 & 1.108 & 0.720 & 8.567 & 30.00 & 5.065 & 0.450 & 31.25 & 17.37 & 180.7 & 160.0 & 108.0 \\
\hline$S / B$ & $10^{-7}$ & $10^{-5}$ & $10^{-6}$ & $10^{-7}$ & $10^{-3}$ & $10^{-4}$ & $10^{-6}$ & $10^{-9}$ & $10^{-6}$ & $10^{-8}$ & $10^{-9}$ & $10^{-8}$ & $10^{-10}$ \\
\hline$E_{o p t}$ & 153.6 & 50.04 & 83.56 & 130.4 & 60.79 & 264.8 & 78.37 & 338.4 & 73.00 & 202.1 & 298.4 & 95.89 & 315.9 \\
\hline
\end{tabular}

Table 1: Parameters and dark matter observables for the benchmark points. The supersymmetric mass spectra are obtained using ISASUGRA 7.51 [12] with the listed input parameters. For all the benchmark points, we assume $A_{0}=0$ and $m_{t}=175 \mathrm{GeV}$. All masses and energies are in GeV. We define the gaugino fraction of the lightest neutralino $\chi$ as $R_{\chi} \equiv\left|Z_{\chi_{1}}\right|^{2}+\left|Z_{\chi_{2}}\right|^{2}$, where $\chi=Z_{\chi_{1}} \tilde{B}+Z_{\chi_{2}} \tilde{W}^{0}+Z_{\chi_{3}} \tilde{H}_{u}^{0}+Z_{\chi_{4}} \tilde{H}_{d}^{0}$. The neutralino relic density $\Omega_{\chi} h^{2}$ is taken from Table 2 of [1], and were calculated using SSARD [13]. The spinindependent (spin-dependent) cross sections on protons $\sigma_{P}^{\mathrm{sc}}\left(\sigma_{P}^{\mathrm{sp}}\right)$ and neutrons $\sigma_{N}^{\mathrm{sc}}\left(\sigma_{N}^{\mathrm{sp}}\right)$ are calculated with Neutdriver [14] and are given in units of $10^{-12} \mathrm{pb}\left(10^{-6} \mathrm{pb}\right)$. The muon fluxes from the Sun $\left(\Phi_{\mu}^{\odot}\right)$ and the Earth $\left(\Phi_{\mu}^{\oplus}\right)$ are in units of $\mathrm{km}^{-2} \mathrm{yr}^{-1}$. The integrated photon fluxes $\Phi_{\gamma}^{1}\left(\Phi_{\gamma}^{50}\right)$ for photon energy threshold $E_{\mathrm{th}}=1 \mathrm{GeV}\left(E_{\mathrm{th}}=50 \mathrm{GeV}\right)$ are in units of $10^{-12} \mathrm{~cm}^{-2} \mathrm{~s}^{-1}\left(10^{-14} \mathrm{~cm}^{-2} \mathrm{~s}^{-1}\right)$. Finally, $S / B$ is the maximal value of the positron signal-to-background ratio, and $E_{\mathrm{opt}}$ is the energy at which this value is realized. 
both spin-independent and spin-dependent matrix elements, the rates for observing muons induced by the collisions in rock of energetic neutrinos produced by relic annihilations inside the Sun and Earth, the rates for photons produced by annihilations in the galactic centre, and the rates for positrons produced by the annihilations of relic particles in the galactic halo. In all cases, we take into account the sensitivities of present and planned detectors in estimating the observability of signals from relic particles. We emphasize that all our results necessarily depend on the halo model used: this is particularly true for the photon signal from the galactic centre. This model-dependence enters when comparing the power of various experimental probes. However, for any given signature, our conclusions concerning the relative ease with which different models can be seen should be quite reliable.

The structure of this paper is as follows. In Sec. 2 we review briefly the experimental constraints that were used as inputs when proposing the benchmark points studied in this paper. In Sec. 3 we compare the predictions of two different codes, Neutdriver [14] and SSARD [13], for direct dark matter detection, obtaining very similar results. We use Neutdriver to calculate muon rates from the Sun and Earth in Sec. 4, and we follow the analysis of [15] to determine the photon and positron rates in Secs. 5 and 6, respectively. Finally, in Sec. 7 we draw some tentative conclusions about the detectability of dark matter particles in the different allowed regions of parameter space, and we contrast the prospects in accelerator and non-accelerator experiments.

\section{Constraints used to Select Benchmark Points}

We restrict our attention to a constrained version of the MSSM (CMSSM) which incorporates a minimal supergravity-inspired model of soft supersymmetry breaking. Universal gaugino masses $m_{1 / 2}$, scalar masses $m_{0}$ (including those of the Higgs multiplets) and trilinear supersymmetry breaking parameters $A_{0}$ are used as inputs at the supersymmetric grand unification scale. In this framework, the Higgs mixing parameter $\mu$ can be derived (up to a sign) from the other MSSM parameters by imposing the electroweak vacuum conditions for any given value of $\tan \beta$. Thus, given the set of input parameters determined by $\left\{m_{1 / 2}, m_{0}, A_{0}, \tan \beta, \operatorname{sgn}(\mu)\right\}$, the entire spectrum of sparticles can be derived. For simplicity, we further restrict our attention to $A_{0}=0$.

The available experimental and phenomenological constraints on the CMSSM parameter space were implemented in [1]. These include the experimental constraints obtained from searches for sparticles [3] and Higgs bosons at LEP [4]. In particular, attention was restricted to parameter choices which guaranteed chargino masses $m_{\chi^{ \pm}}>103.5 \mathrm{GeV}$ [16] and selectron 
masses $m_{\tilde{e}}>99.4 \mathrm{GeV}$ [17]. The lower limit on the mass of a Standard Model Higgs boson imposed by the combined LEP experiments is $113.5 \mathrm{GeV}$ [4], and this limit also applies to the lightest supersymmetric Higgs boson $h$ in the CMSSM. To calculate $m_{h}$ theoretically, we use the FeynHiggs code [18], which includes one-loop effects and also the leading two-loop contributions. To account for uncertainties in theoretical calculations of $m_{h}$ [18], for any given value of $m_{t}$, we restrict our CMSSM parameter choices to those yielding $m_{h} \geq 113 \mathrm{GeV}$. In addition, the theoretical value of $m_{h}$ in the MSSM is quite sensitive to $m_{t}$, the pole mass of the top quark: we use $m_{t}=175 \mathrm{GeV}$ as default. All but one of the benchmark points satisfy $m_{h}>113 \mathrm{GeV}$. In view of the expected accuracy $\sim 3 \mathrm{GeV}$ of the FeynHiggs code, we consider that all the proposed points are compatible with the LEP lower limit of $113.5 \mathrm{GeV}[4]$.

We also compute the rate for $b \rightarrow s \gamma$ decay and compare it with the experimental range [5]. We implement the new NLO $b \rightarrow s \gamma$ calculations of [19] when $\tilde{M}>500 \mathrm{GeV}$, where $\tilde{M}=\min \left(m_{\tilde{q}}, m_{\tilde{g}}\right)$. Otherwise, we use only the LO calculations and assign a larger theoretical error. For the experimental value, we combine the CLEO measurement with the recent BELLE result $[5], \mathcal{B}(b \rightarrow s \gamma)=(3.21 \pm 0.44 \pm 0.26) \times 10^{-4}$. In our implementation, we allow CMSSM parameter choices that, after including the theoretical errors $\sigma_{t h}$ due to the scale and model dependences, may fall within the $95 \%$ confidence level range $2.33 \times 10^{-4}<$ $\mathcal{B}(b \rightarrow s \gamma)<4.15 \times 10^{-4}$.

The final experimental contraint we consider is the $g_{\mu}-2$ value reported by the BNL E821 experiment [6]. This experiment has found an apparent discrepancy with the Standard Model prediction at the level of $2.6 \sigma: \delta a_{\mu}=(43 \pm 16) \times 10^{-10}$. A large number of theoretical papers have discussed the interpretation of the BNL measurement within supersymmetry [20, 21], and they generally agree that $\mu>0$ is favoured by the BNL measurement. The calculations we use in this paper are taken from [21], which are based on [22], including also the leading two-loop electroweak correction factor [23].

We assume that $R$ parity is conserved, and that the stable LSP is the lightest neutralino $\chi$ [24]. We then constrain the CMSSM parameter space by requiring the calculated supersymmetric relic density to fall within the range $0.1<\Omega_{\chi} h^{2}<0.3$ preferred by astrophysics and cosmology. The upper limit on $\Omega_{\chi} h^{2}$ is conservative, being based only on the lower limit on the age of the Universe of 12 Gyr. Smaller values of $\Omega_{\chi} h^{2}$ are certainly possible, since some of the cold dark matter might not consist of LSPs. However, allowing smaller values of $\Omega_{\chi} h^{2}$ would open up only a very small extra region of the $\left(m_{0}, m_{1 / 2}\right)$ plane.

Good overall consistency was found in [1] between these relic density calculations, the LEP and other sparticle mass limits, the LEP Higgs limit, measurements of $b \rightarrow s \gamma$ and the 
recent BNL measurement of $g_{\mu}-2$, if $\mu>0$ and $\tan \beta \gtrsim 5$. For $\tan \beta \gtrsim 50$, there are not substantial regions with consistent electroweak vacua.

The values of the CMSSM parameters for the benchmark points are shown in Table 1. From these, soft masses are determined with ISASUGRA 7.51, and relic densities are calculated with a recent analysis [11] using SSARD that extends previous results [2] to larger $\tan \beta>20$. The chosen values of $\tan \beta$ range from 5 to about 50 . In deference to $g_{\mu}-2$, most of the points proposed have $\mu>0$, but only about a half of the chosen points yield values of $g_{\mu}-2$ within $2 \sigma$ of the present central experimental value, and two of the points have $\mu<0$. The amount of CMSSM parameter fine-tuning required for electroweak symmetry breaking, along with the sensitivity of the relic density to the precise values of the input CMSSM parameters, are given in [1] together with the corresponding sparticle spectra.

\section{Direct Detection via Elastic Scattering}

The prospects for direct detection of neutralinos can be reduced to the computation of the neutralino-proton elastic scattering cross section. We first review the ingredients of this calculation that are implemented in SSARD. The MSSM Lagrangian leads to the following low-energy effective four-fermion Lagrangian suitable for describing elastic $\chi$-nucleon scattering [25]:

$$
\begin{aligned}
\mathcal{L} & =\bar{\chi} \gamma^{\mu} \gamma^{5} \chi \bar{q}_{i} \gamma_{\mu}\left(\alpha_{1 i}+\alpha_{2 i} \gamma^{5}\right) q_{i}+\alpha_{3 i} \bar{\chi} \chi \bar{q}_{i} q_{i} \\
& +\alpha_{4 i} \bar{\chi} \gamma^{5} \chi \bar{q}_{i} \gamma^{5} q_{i}+\alpha_{5 i} \bar{\chi} \chi \bar{q}_{i} \gamma^{5} q_{i}+\alpha_{6 i} \bar{\chi} \gamma^{5} \chi \bar{q}_{i} q_{i}
\end{aligned}
$$

This Lagrangian is to be summed over the quark generations, and the subscript $i$ labels uptype quarks $(i=1)$ and down-type quarks $(i=2)$. The terms with coefficients $\alpha_{1 i}, \alpha_{4 i}, \alpha_{5 i}$ and $\alpha_{6 i}$ are velocity-dependent contributions and may be neglected for the purpose of direct detection calculations. The coefficients relevant for our discussion are, then, the spinindependent or scalar coefficients

$$
\begin{aligned}
\alpha_{3 i}= & -\frac{1}{2\left(m_{1 i}^{2}-m_{\chi}^{2}\right)} \operatorname{Re}\left[\left(X_{i}\right)\left(Y_{i}\right)^{*}\right]-\frac{1}{2\left(m_{2 i}^{2}-m_{\chi}^{2}\right)} \operatorname{Re}\left[\left(W_{i}\right)\left(V_{i}\right)^{*}\right] \\
& -\frac{g m_{q i}}{4 m_{W} B_{i}}\left[\operatorname{Re}\left(\delta_{1 i}\left[g Z_{\chi_{2}}-g^{\prime} Z_{\chi_{1}}\right]\right) D_{i} C_{i}\left(-\frac{1}{m_{H_{1}}^{2}}+\frac{1}{m_{H_{2}}^{2}}\right)\right. \\
& \left.+\operatorname{Re}\left(\delta_{2 i}\left[g Z_{\chi_{2}}-g^{\prime} Z_{\chi_{1}}\right]\right)\left(\frac{D_{i}^{2}}{m_{H_{2}}^{2}}+\frac{C_{i}^{2}}{m_{H_{1}}^{2}}\right)\right]
\end{aligned}
$$

and the spin-dependent coefficients

$$
\alpha_{2 i}=\frac{1}{4\left(m_{1 i}^{2}-m_{\chi}^{2}\right)}\left[\left|Y_{i}\right|^{2}+\left|X_{i}\right|^{2}\right]+\frac{1}{4\left(m_{2 i}^{2}-m_{\chi}^{2}\right)}\left[\left|V_{i}\right|^{2}+\left|W_{i}\right|^{2}\right]
$$




$$
-\frac{g^{2}}{4 m_{Z}^{2} \cos ^{2} \theta_{W}}\left[\left|Z_{\chi_{3}}\right|^{2}-\left|Z_{\chi_{4}}\right|^{2}\right] \frac{T_{3 i}}{2} .
$$

Here $m_{1 i}$ and $m_{2 i}$ are the squark mass eigenvalues,

$$
\begin{aligned}
X_{i} & \equiv \eta_{11}^{i *} \frac{g m_{q_{i}} Z_{\chi_{5-i}}^{*}}{2 m_{W} B_{i}}-\eta_{12}^{i *} e_{i} g^{\prime} Z_{\chi_{1}}^{*} \\
Y_{i} & \equiv \eta_{11}^{i *}\left(\frac{y_{i}}{2} g^{\prime} Z_{\chi_{1}}+g T_{3 i} Z_{\chi_{2}}\right)+\eta_{12}^{i *} \frac{g m_{q_{i}} Z_{\chi_{5-i}}}{2 m_{W} B_{i}} \\
W_{i} & \equiv \eta_{21}^{i *} \frac{g m_{q_{i}} Z_{\chi_{5-i}}^{*}}{2 m_{W} B_{i}}-\eta_{22}^{i *} e_{i} g^{\prime} Z_{\chi_{1}}^{*} \\
V_{i} & \equiv \eta_{22}^{i *} \frac{g m_{q_{i}} Z_{\chi_{5-i}}}{2 m_{W} B_{i}}+\eta_{21}^{i *}\left(\frac{y_{i}}{2} g^{\prime} Z_{\chi_{1}}+g T_{3 i} Z_{\chi_{2}}\right),
\end{aligned}
$$

and the coefficients $Z_{\chi_{i}}$ define the composition of the lightest neutralino through

$$
\chi=Z_{\chi_{1}} \tilde{B}+Z_{\chi_{2}} \tilde{W}+Z_{\chi_{3}} \tilde{H}_{1}+Z_{\chi_{4}} \tilde{H}_{2}
$$

The parameters $e_{i}, T_{3 i}, y_{i}$ denote electric charge, isospin and hypercharge (normalized so that $\left.e_{i}=T_{3 i}+\frac{y_{i}}{2}\right)$, respectively, and

$$
\begin{array}{cl}
\delta_{1 i}=\left(Z_{\chi_{3}}, Z_{\chi_{4}}\right) & \delta_{2 i}=\left(Z_{\chi_{4}},-Z_{\chi_{3}}\right) \\
B_{i}=(\sin \beta, \cos \beta) & A_{i}=(\cos \beta,-\sin \beta) \\
C_{i}=(\sin \alpha, \cos \alpha) & D_{i}=(\cos \alpha,-\sin \alpha)
\end{array}
$$

for (up, down) type quarks. We denote by $m_{H_{2}}<m_{H_{1}}$ the two scalar Higgs masses, and $\alpha$ is the Higgs mixing angle. Finally, $\eta_{j k}^{i}$ are elements of the matrix that diagonalizes squark mass matrices through $\operatorname{diag}\left(m_{1 i}^{2}, m_{2 i}^{2}\right) \equiv \eta^{i} M_{i}^{2}\left(\eta^{i}\right)^{-1}$.

The spin-independent (scalar) part of the cross section can be written as

$$
\sigma_{3}=\frac{4 m_{r}^{2}}{\pi}\left[Z f_{p}+(A-Z) f_{n}\right]^{2}
$$

where $m_{r}$ is the reduced neutralino mass and $A, Z$ are the atomic number and nuclear electric charge,

$$
\frac{f_{p}}{m_{p}}=\sum_{q=u, d, s} f_{T q}^{(p)} \frac{\alpha_{3 q}}{m_{q}}+\frac{2}{27} f_{T G}^{(p)} \sum_{c, b, t} \frac{\alpha_{3 q}}{m_{q}}
$$

where $m_{p}$ is the proton mass, and $f_{n}$ is defined similarly. The parameters $f_{T q}^{(p)}$ are defined by

$$
m_{p} f_{T q}^{(p)} \equiv\left\langle p\left|m_{q} \bar{q} q\right| p\right\rangle \equiv m_{q} B_{q}
$$


while $f_{T G}^{(p)}=1-\sum_{q=u, d, s} f_{T q}^{(p)}[26]$. Following the analysis in [27, 28] we use the following values of $f_{T q}^{(p)}$ :

$$
\begin{aligned}
& f_{T u}^{(p)}=0.020 \pm 0.004, \quad f_{T d}^{(p)}=0.026 \pm 0.005 \\
& f_{T s}^{(p)}=0.118 \pm 0.062,
\end{aligned}
$$

where essentially all the error in $f_{T s}^{(p)}$ arises from the uncertainty in strangeness composition

$$
y \equiv \frac{2 B_{s}}{B_{d}+B_{u}}=0.2 \pm 0.1
$$

The corresponding values for the neutron are

$$
\begin{aligned}
f_{T u}^{(n)} & =0.014 \pm 0.003, \quad f_{T d}^{(n)}=0.036 \pm 0.008 \\
f_{T s}^{(n)} & =0.118 \pm 0.062 .
\end{aligned}
$$

These values are based in part on the experimental value of the $\pi$-nucleon $\sigma$ term [29]

$$
\sigma \equiv \frac{1}{2}\left(m_{u}+m_{d}\right) \times\left(B_{d}+B_{u}\right)=45 \pm 8 \mathrm{MeV} .
$$

The larger value of $\sigma=65 \mathrm{MeV}$ [30] considered by [31] leads to scattering cross section which are larger by a factor of about 3. It is clear already that the difference between the scalar parts of the cross sections for scattering off protons and neutrons must be rather small.

The spin-dependent part of the elastic $\chi$-nucleus cross section can be written as

$$
\sigma_{2}=\frac{32}{\pi} G_{F}^{2} m_{r}^{2} \Lambda^{2} J(J+1)
$$

where $G_{F}$ is the Fermi constant, $m_{r}$ is again the reduced neutralino mass, $J$ is the spin of the nucleus, and

$$
\Lambda \equiv \frac{1}{J}\left(a_{p}\left\langle S_{p}\right\rangle+a_{n}\left\langle S_{n}\right\rangle\right)
$$

where $\left\langle S_{p, n}\right\rangle$ are expectation values of the spin content of the nucleus and

$$
a_{p}=\sum_{i} \frac{\alpha_{2 i}}{\sqrt{2} G_{F}} \Delta_{i}^{(p)}, \quad a_{n}=\sum_{i} \frac{\alpha_{2 i}}{\sqrt{2} G_{F}} \Delta_{i}^{(n)}
$$

The factors $\Delta_{i}^{(p, n)}$ parameterize the quark spin content of the nucleon. A recent global analysis of QCD sum rules for the $g_{1}$ structure functions [32], including $\mathcal{O}\left(\alpha_{s}^{3}\right)$ corrections, corresponds formally to the values

$$
\begin{aligned}
& \Delta_{u}^{(p)}=0.78 \pm 0.02, \quad \Delta_{d}^{(p)}=-0.48 \pm 0.02 \\
& \Delta_{s}^{(p)}=-0.15 \pm 0.02 .
\end{aligned}
$$


In the case of the neutron, we have $\Delta_{u}^{(n)}=\Delta_{d}^{(p)}, \Delta_{d}^{(n)}=\Delta_{u}^{(p)}$, and $\Delta_{s}^{(n)}=\Delta_{s}^{(p)}$.

The calculation of the neutralino-nucleon elastic scattering cross-sections in Neutdriver is based on [33]. The calculation of the spin-dependent contribution is identical to the one presented above. However, the spin-independent computation contains several additional pieces. First, the heavy flavor squark contribution is treated in exact one-loop approximation as in [33], and (8) is replaced by

$$
\frac{f_{p}}{m_{p}}=\sum_{q=u, d, s} f_{T q}^{(p)} \frac{\alpha_{3 q}^{\tilde{q}}}{m_{q}}-\frac{8 \pi}{9 \alpha_{S}} f_{T G}^{(p)}\left[B_{D}-\frac{m_{\chi}^{2}}{4} B_{1 D}\right]
$$

where $B_{D}$ and $B_{1 D}$ are loop integrals defined in Eqs. (18a) and (18c) of [33], respectively. Second, Neutdriver also includes a Higgs contribution through squark loops (see the last term in Eq. (43) of [33]). Finally, it includes several contributions from twist-2 operators, which are listed in Eq. (46) of [33].

Numerical values from Neutdriver for the spin-independent and the spin-dependent components of the elastic cross sections for the scattering of neutralinos on protons and neutrons for each of the benchmark points are presented in Table 1. (For other recent work in the CMSSM, see, e.g., [34].) In Fig. 2, we compare the results for the spin-independent $\sigma_{P}^{s c}$ and spin-dependent $\sigma_{P}^{s p}$ cross-sections for neutralino-proton and neutralino-neutron scattering using SSARD [13] and Neutdriver [14]. (For the latter, we have changed the default values of the quantities $f_{T q}^{(p)}, f_{T q}^{(n)}, \Delta_{q}^{(p)}$, and $\Delta_{q}^{(n)}$ to match those in (10), (12) and (17).) The differences are insignificant relative to the effects of different choices of CMSSM model parameters. Recall also that the mass spectra outputs of SSARD and ISASUGRA differ, as may be seen by comparing Tables 1 and 3 of [1]. Fig. 2 shows the projected sensitivities (a,b) for CDMS II [35] and CRESST [36] (solid) and GENIUS [37] (dashed), and (c) a $100 \mathrm{~kg}$ NAIAD [38] detector, as well as (d) the existing DAMA limit [39]. Obtaining a competitive limit for the spin-dependent scattering on a neutron in the latter case might be possible with a large ${ }^{73} \mathrm{Ge}$ or Xenon detector.

As was found in [27], there are strong cancellations in the spin-independent cross sections when $\mu<0$. These cancellations are due to sign differences between the up- and down-type quark contributions to the Higgs exchange terms in $\alpha_{3}$ in (2). Nominally, these cancellations occur only for a specific range in the neutralino mass. For $\tan \beta=10$, the cancellations occur for $m_{\chi} \simeq 150-350 \mathrm{GeV}$, and are particularly effective when $m_{\chi} \simeq 200-250 \mathrm{GeV}$. As one can see in Table 1 and Fig. 2, point D falls exactly into this range, thus explaining why its scalar cross section is anomalously small. Similarly, for $\tan \beta=35$, there are strong cancellations at $m_{\chi} \simeq 400-600 \mathrm{GeV}$ [40]. Unfortunately, point $\mathrm{K}$ happens to fall in this range as well. Thus 

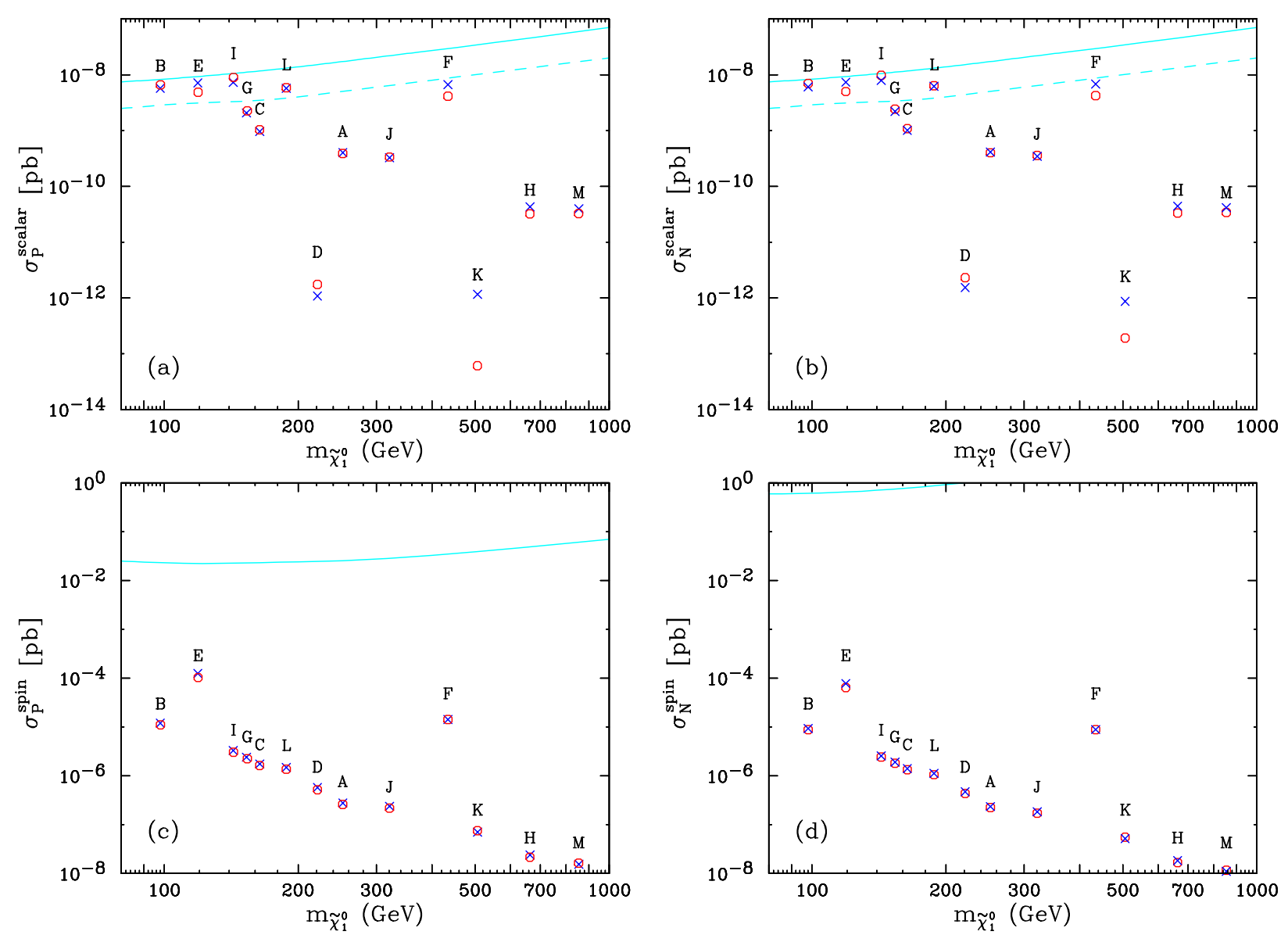

Figure 2: Elastic cross sections for ( $a, b)$ spin-independent scattering and (c,d) spindependent scattering on $(a, c)$ protons and $(b, d)$ neutrons. The predictions of SSARD (blue crosses) and Neutdriver (red circles) for neutralino-nucleon scattering are compared. Projected sensitivities (a,b) for CDMS II [35] and CRESST [36] (solid) and GENIUS [37] (dashed) and (c) for a $100 \mathrm{~kg} N A I A D$ array [38], as well as (d) the existing DAMA limit [39] are also shown.

the two benchmark points with $\mu<0$ are predicted to have very small spin-independent cross sections, but this would not generally be true for other CMSSM models with $\mu<0$. As one might expect, the differences between the SSARD and Neutdriver codes are largest for these points that exhibit delicate cancellations.

Comparing the benchmark model predictions with the projected sensitivities, we see that spin-independent scattering seems to offer the best prospects for direct detection. Among the proposed benchmark points, models I, B, E, L, G, F, and C seem to offer the best detection prospects. In particular, the first four of these models would apparently be detectable with the proposed GENIUS detector. 


\section{Neutrinos from Annihilations in the Sun and Earth}

Dark matter particles collect in the gravitational wells at the centers of astrophysical bodies, leading to large densities and enhanced pair annihilation rates. While most annihilation products are immediately trapped or absorbed, neutrinos may propagate for long distances and be detected near the Earth's surface through their charged-current conversion to muons. High-energy muons produced by neutrinos from the centers of the Sun [41] and Earth [42] are therefore prominent signals for indirect dark matter detection.

The muon detection rate is dependent on both the neutralino annihilation rate and the resulting neutrino energy spectrum. The neutralino annihilation rate is proportional to the present dark matter density at the core of the Sun or Earth. Determinations of these densities are involved, but well understood. Various aspects of these calculations are reviewed in [14], and estimates of neutralino annihilation rates in the CMSSM for both the Sun and the Earth are given in [15]. (For other recent work in the CMSSM, see, e.g., [43, 44].) For the Sun, the annihilation rate has typically reached equilibrium and decreases for increasing neutralino mass.

The neutrino energy spectrum depends on the neutralino composition. Neutralinos annihilate primarily to fermion pairs and gauge boson pairs. Annihilation to fermion pairs is helicity-suppressed, and so is significant only for heavy fermions, such as $b$ quarks and $\tau$ leptons, and $t$ quarks if kinematically allowed. Neutrinos from these decays are typically rather soft. Annihilation to gauge bosons is possible only for neutralinos that are heavier than $W$ bosons and have a significant Higgsino component. When possible, however, these annihilation channels typically dominate, producing hard neutrinos from two-body gauge boson decay. In this case, the muon flux is greatly enhanced, as both the cross section for conversion to muons and the muon range are proportional to the neutrino energy.

Muon fluxes for each of the benchmark points are given in Fig. 3, using Neutdriver with a fixed constant local density $\rho_{0}=0.3 \mathrm{GeV} / \mathrm{cm}^{3}$ and neutralino velocity dispersion $\bar{v}=270 \mathrm{~km} / \mathrm{s}$. For the points considered, rates from the Sun are far more promising than rates from the Earth. For the Sun, muon fluxes are for the most part anti-correlated with neutralino mass for the reason noted above. There are two strong exceptions, however: the focus point models $\mathrm{E}$ and $\mathrm{F}$ have anomalously large fluxes. In these cases, the dark matter's Higgsino content, though still small, is significant (see Table 1), leading to annihilations to gauge boson pairs, hard neutrinos, and enhanced detection rates, as discussed above.

The potentials of current and planned neutrino telescopes have been reviewed in [15]. The exact reach depends on the salient features of a particular detector, e.g., its physical 

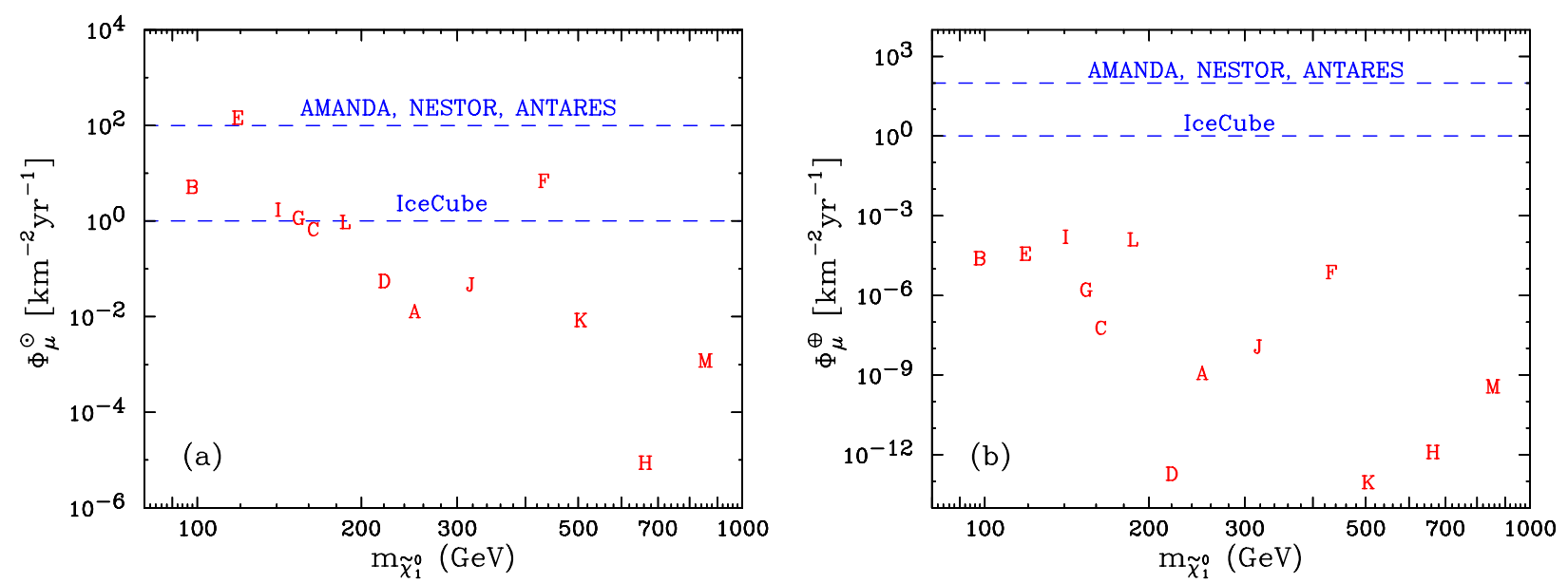

Figure 3: Muon fluxes from neutrinos originating from relic annihilations inside (a) the Sun and (b) the Earth. Approximate sensitivities of near future neutrino telescopes $\left(\Phi_{\mu}=10^{2} \mathrm{~km}^{-2} \mathrm{yr}^{-1}\right.$ for AMANDA II [45], NESTOR [46], and ANTARES [47], and $\Phi_{\mu}=1 \mathrm{~km}^{-2} \mathrm{yr}^{-1}$ for IceCube [48]) are also indicated.

dimensions and muon energy threshold, and the expected characteristics of the signal, e.g., its angular dispersion, energy spectrum and source (Sun or Earth). Two sensitivities, which are roughly indicative of the potential of upcoming neutrino telescope experiments, are given in Fig. 3. For focus-point model E, where the neutralino is both light and significantly different from pure Bino-like, detection in the near future at AMANDA II [45], NESTOR [46], and ANTARES [47] is possible. Point F may be within reach of IceCube [48], as the neutralino's significant Higgsino component compensates for its large mass. For point B, and possibly also points I, G, C, and L, the neutralino is nearly pure Bino, but is sufficiently light that detection at IceCube may also be possible.

Muon energy thresholds specific to individual detectors have not been included. For AMANDA II and, especially, IceCube, these thresholds may be large, significantly suppressing the muon signal in models with $m_{\chi}$ less than about 4 to $6 E_{\mu}^{\text {th }}[49,44]$. Note also that, for certain neutralino masses and properties, a population of dark matter particles in solar system orbits may boost the rates presented here by up to two orders of magnitude [50]. While this effect deserves further study, here we have conservatively neglected this possible enhancement. 


\section{Photons from Annihilations in the Galactic Center}

As with the centers of the Sun and Earth, the center of the galaxy may attract a significant overabundance of relic dark matter particles [51]. Relic pair annihilation at the galactic center will then produce an excess of photons, which may be observed in gamma ray detectors. While monoenergetic signals from $\chi \chi \rightarrow \gamma \gamma$ and $\chi \chi \rightarrow \gamma Z$ would be spectacular [52], they are loop-suppressed and unobservable for these benchmark points. We therefore consider continuum photon signals here.

The integrated photon flux above some photon energy threshold $E_{\text {th }}$ is [15]

$$
\Phi_{\gamma}\left(E_{\mathrm{th}}\right)=5.6 \times 10^{-10} \mathrm{~cm}^{-2} \mathrm{~s}^{-1} \times \sum_{i} \int_{E_{\mathrm{th}}}^{m_{\chi}} d E \frac{d N_{\gamma}^{i}}{d E}\left(\frac{\sigma_{i} v}{\mathrm{pb}}\right)\left(\frac{100 \mathrm{GeV}}{m_{\chi}}\right)^{2} \bar{J}(\Delta \Omega) \Delta \Omega
$$

where the sum is over all annihilation channels $i, d N_{\gamma}^{i} / d E$ is the differential gamma ray multiplicity for process $i, \Delta \Omega$ is the solid angle of the field of view of a given telescope, and $\bar{J}$ is a measure of the cuspiness of the galactic halo density profile. There is a great deal of uncertainty in $\bar{J}$, with possible values in the range 3 to $10^{5}[52]$.

The integrated photon flux $\Phi\left(E_{\mathrm{th}}\right)$ is given in Fig. 4 for each of the benchmark points. We choose $\Delta \Omega=10^{-3}$ and a moderate value of $\bar{J}=500$. Estimates for point source flux sensitivities of several gamma ray detectors, both current and planned, are also shown. The space-based detectors EGRET, AMS/ $\gamma$ and GLAST can detect soft photons, but are limited in flux sensitivity by their small effective areas. Ground-based telescopes, such as MAGIC, HESS, CANGAROO and VERITAS, are much larger and so sensitive to lower fluxes, but are limited by higher energy thresholds. These sensitivities are not strictly valid for observations of the galactic center. Nevertheless, they provide rough guidelines for what sensitivities may be expected in coming years. For a discussion of these estimates, their derivation, and references to the original literature, see [15].

Integrated fluxes for the benchmark points are given in Fig. 5 for two representative energy thresholds: $1 \mathrm{GeV}$, accessible to space-based detectors, and $50 \mathrm{GeV}$, characteristic of ground-based telescopes. Estimated sensitivities for two of the more promising experiments, GLAST [53] and MAGIC [54], are also shown. From (19), we expect the photon flux to be inversely correlated with neutralino mass. Roughly speaking, this general trend is seen in Fig. 5a. For Fig. 5b, it is offset by the requirement of a hard photon, which suppresses the signal from light neutralinos. In both cases, however, this general trend may be disrupted by a variety of additional effects. In particular, the photon spectrum is relatively hard for annihilation to gauge bosons; $\Phi\left(E_{\mathrm{th}}\right)$ is, then, enhanced for the focus point models $\mathrm{E}$ and $\mathrm{F}$, which have neutralinos with significant Higgsino components. The cross section $\sigma_{i}$ for 


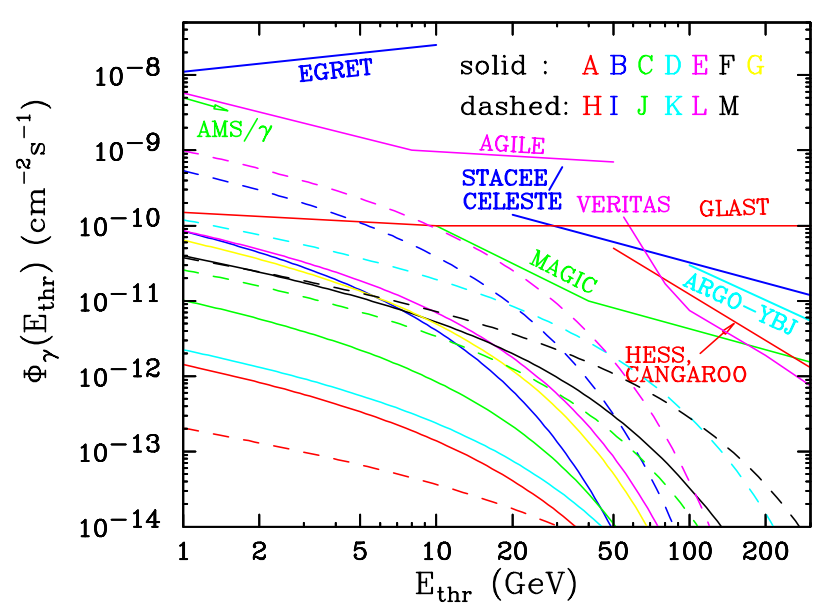

Figure 4: The integrated photon flux $\Phi\left(E_{\mathrm{th}}\right)$ as a function of photon energy threshold $E_{\text {th }}$ for photons produced by relic annihilations in the galactic center. A moderate halo parameter $\bar{J}=500$ is assumed. Point source flux sensitivities for various gamma ray detectors are also shown.

annihilation to $b \bar{b}$ through $s$-channel pseudoscalar Higgs is also enhanced for large $\tan \beta[8]$, boosting photon signals at points I, J, K, L, and M.

GLAST appears to be particularly promising, with points I and L giving observable signals. Recall, however, that all predicted fluxes scale linearly with $\bar{J}$. For isothermal halo density profiles, the fluxes may be reduced by two orders of magnitude. On the other hand, for particularly cuspy halo models, such as those in [55], all fluxes may be enhanced by two orders of magnitude, leading to detectable signals in GLAST for almost all points, and at MAGIC for the majority of benchmark points.

\section{Positrons from Annihilations in the Galactic Halo}

Relic neutralino annihilations in the galactic halo may also be detected through positron excesses in space-based and balloon experiments $[56,57]$. The positron flux may be written as $[57]$

$$
\frac{d \Phi_{e^{+}}}{d \Omega d E}=\frac{\rho^{2}}{m_{\chi}^{2}} \sum_{i} \sigma_{i} v B_{e^{+}}^{i} \int d E_{0} f_{i}\left(E_{0}\right) G\left(E_{0}, E\right),
$$

where $\rho$ is the local neutralino mass density, the sum is over all annihilation channels $i$, and $B_{e^{+}}^{i}$ is the branching fraction to positrons in channel $i$. The initial positron energy distribution is given by the source function $f\left(E_{0}\right)$, and the Green function $G\left(E_{0}, E\right)$ propagates positrons in the galaxy. We use the Green function corresponding to a modified isothermal halo with size $4 \mathrm{kpc}$ given in [57]. The differential positron fluxes for the benchmark points 

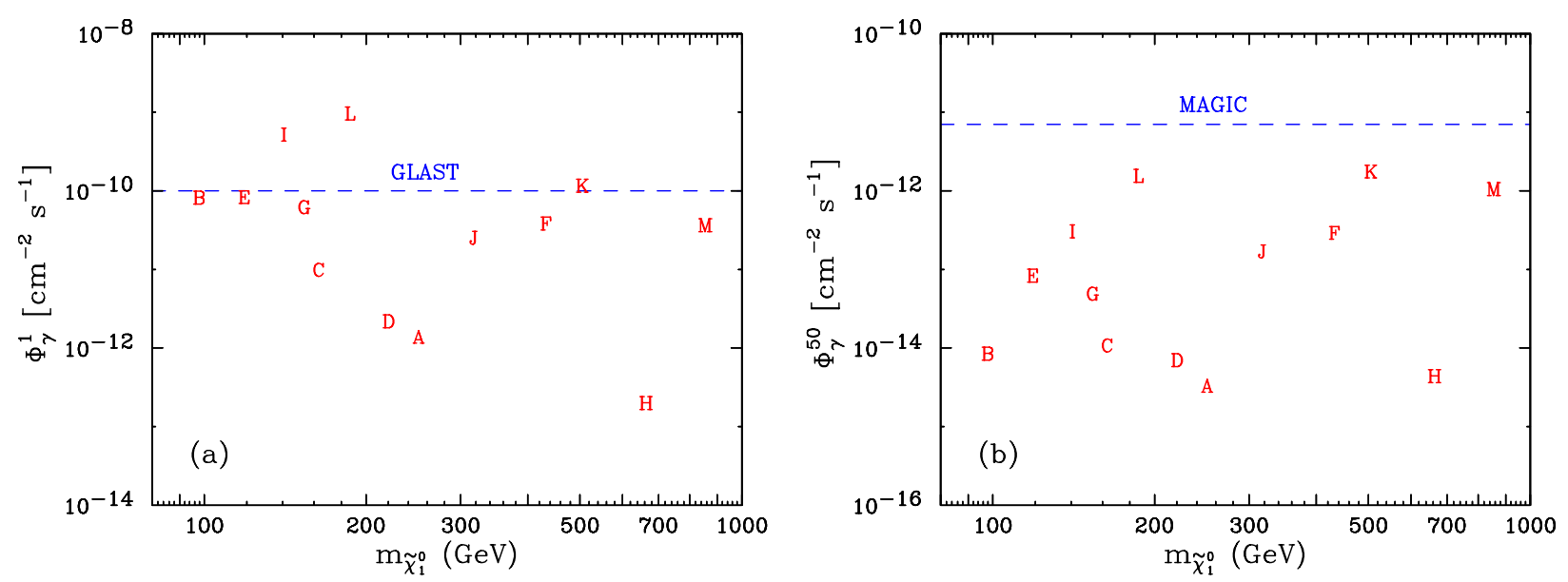

Figure 5: Comparisons between predicted integrated fluxes and prospective experimental sensitivities for photons with (a) a 1 GeV threshold, and (b) a 50 GeV threshold, following [15]. Estimated sensitivities for (a) GLAST [53] and (b) MAGIC [54] are also shown. A moderate halo parameter $\bar{J}=500$ is assumed.

are given in Fig. 6. Note that the background spectrum drops rapidly with energy; hard positrons from neutralino annihilation are most easily observed.

To estimate the observability of a positron excess, we follow the procedure advocated in [15]. For each benchmark spectrum, we find the positron energy $E_{\text {opt }}$ at which the positron signal to background ratio $S / B$ is maximized. For detection, we then require that $S / B$ at $E_{\text {opt }}$ be above some value. The sensitivities of a variety of experiments have been estimated in [15]. Among these experiments, the most promising is AMS [58], the anti-matter detector to be placed on the International Space Station. AMS will detect unprecedented numbers of positrons in a wide energy range. We estimate that a $1 \%$ excess in an fairly narrow energy bin, as is characteristic of the neutralino signal, will be statistically significant.

Estimates of $E_{\text {opt }}$ and the maximal $S / B$ for each benchmark point are given in Fig. 7 . To an excellent approximation, energetic positrons are produced only when neutralinos annihilate to gauge bosons that decay directly to positrons. Because this decay is two-body, $E_{\text {opt }} \approx m_{\chi} / 2$ for all benchmark points. As expected from (20), $S / B$ is typically inversely correlated with neutralino mass. As discussed in Sec. 4 for the case of neutrinos, however, there are two strong exceptions: the focus point models E and F. Again, these points have mixed gaugino-Higgsino dark matter. Rates for annihilation to gauge bosons and, consequently, the positron signals are therefore greatly enhanced.

Even for points E and F, however, discovery of the positron excess is challenging for the smooth isothermal halo considered here. The positron search is most effective for light 


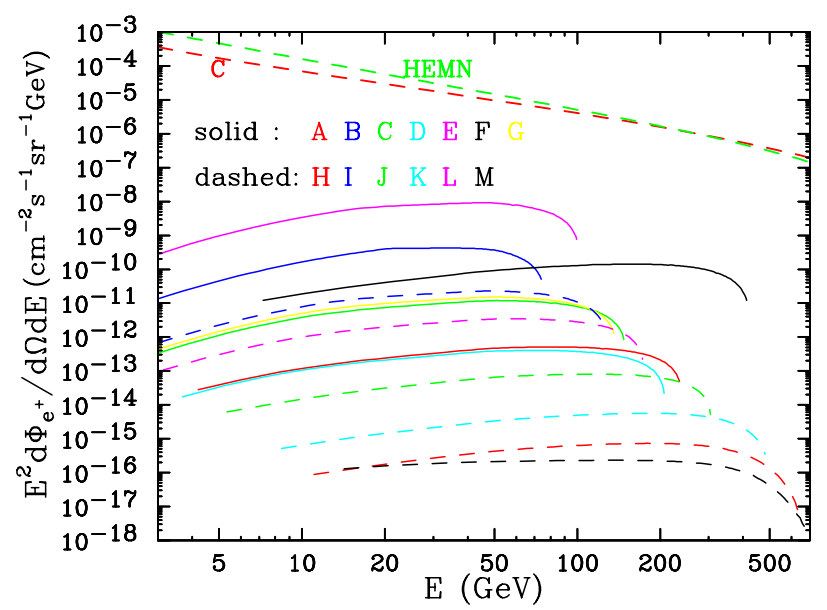

Figure 6: Differential positron fluxes produced by relic annihilations in the galactic halo. Background fluxes are also shown for two models from [57].

neutralinos that are more Higgsino-like than those represented in this set of benchmark points. However, as with the photon signal, positron rates are sensitive to the halo model assumed; for clumpy halos [59], the rate may be enhanced by orders of magnitude [57].

\section{Conclusions}

In this paper, we have provided indicative estimates of the rates that could be expected for the benchmark supersymmetric scenarios proposed in [1]. We emphasize that, in addition to the supersymmetric model dependences of these calculations, there are important astrophysical uncertainties. These include the overall halo density, the possibility that it may be enhanced in the solar system, its cuspiness near the galactic centre, and its clumpiness elsewhere. For these reasons, our conclusions about the relative ease with which different models may be detected using the same signature may be more reliable than the absolute strengths we predict, or comparisons between the observabilities of different signatures. Nevertheless, our estimates do indicate that there may be good prospects for astrophysical detection of quite a large number of the benchmark scenarios.

In particular, the direct detection of relic particles by spin-independent elastic scattering in models I, B, E and L may be possible using the projected GENIUS [37] detector, with models G, F and C not far from the likely threshold of detectability. The prospects of detecting spin-dependent elastic scattering do not, however, look so promising in the benchmark scenarios studied. The indirect detection of muons generated by high-energy neutrinos due to annihilations inside the Sun should be most easily detectable in models E, F and 

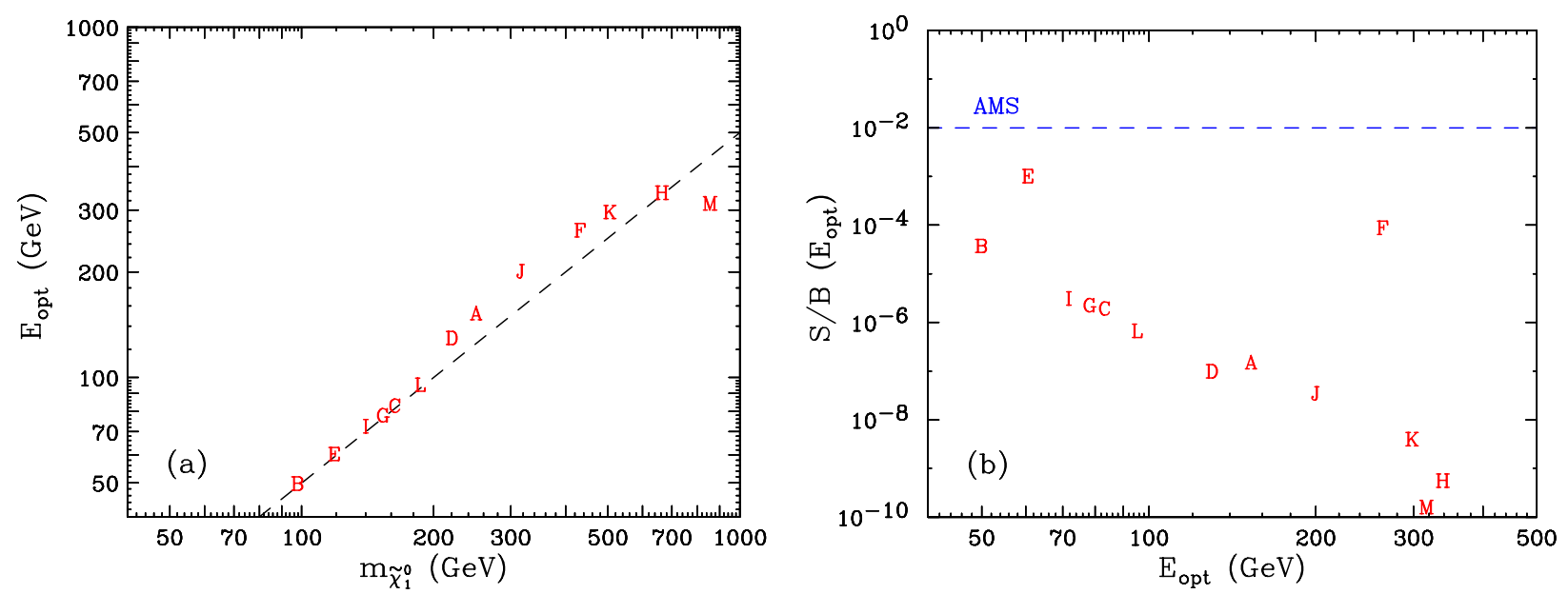

Figure 7: (a) The optimal energies for which the positron signal to background ratios $S / B$ are maximized, and (b) $S / B$ at these energies for each of the benchmark points, following [15]. In (a), the dashed line is for $E_{\mathrm{opt}}=m_{\chi} / 2$, and in (b), the estimated sensitivity of the AMS [58] experiment is shown.

B, followed by models I, G, L and C, which offer prospects with the proposed IceCube [48] detector. However, unless there is a substantial solar-system enhancement, the prospects for detecting annihilations inside the Earth are not so encouraging. Models L and I offer the best prospects for the detection of photons from annihilations in the galactic centre, followed by models K, B, E and G. Here the best prospects may be those for the GLAST [53] satellite, with its relatively low threshold. However, there may also be prospects for ground-based experiments such as MAGIC [54], if the halo is cuspier at the galactic centre than we have assumed. Models E, F and B offer the positron signals with the largest signal-to-background ratios, though apparently requiring a sensitivity greater than that expected for AMS [58], unless the halo is rather clumpy.

In specifying the benchmark models, the constraint coming from the anomalous magnetic moment of the muon was not imposed rigorously. However, it was noted that the more $g_{\mu}-2$-friendly models I, L, B, G, C and J offered good prospects for detecting several supersymmetric particles at the LHC and/or a linear $e^{+} e^{-}$collider with $1 \mathrm{TeV}$ in the centre of mass. Most of these models also exhibit good prospects for dark matter detection, with the exception of model J. Among the less $g_{\mu}-2$-friendly models, we note that $\mathrm{E}, \mathrm{F}$ and $\mathrm{K}$ offer some astrophysical prospects. This is particularly interesting in the case of focus point model $\mathrm{F}$, which does not offer generous prospects at colliders, and model $\mathrm{K}$, which is not easy to explore with a linear $e^{+} e^{-}$collider. On the other hand, models $\mathrm{M}$ and $\mathrm{H}$, which are particularly difficult to explore with colliders, also do not offer bright prospects 
for astrophysical detection.

Our analysis indicates the effort required to cover the possible supersymmetric parameter space via a number of different astrophysical signatures, at least within the CMSSM assumptions used here. It would be interesting to extend such a benchmark analysis to other types of supersymmetric models, but that lies beyond the scope of this paper. Ultimately, one would hope to be able to confront accelerator and astrophysical measurements of supersymmetry, and make non-trivial cross-checks of our CMSSM assumptions, but that is for the future. For the moment, the race to discover supersymmetry is still open, and our analysis indicates that there may be good prospects for detecting supersymmetric dark matter before the LHC comes into operation.

\section{Acknowledgments}

The work of J.L.F. was supported in part by the US Department of Energy under cooperative research agreement DF-FC02-94ER40818. The work of K.A.O. was supported partly by DOE grant DE-FG02-94ER-40823. K.T.M. thanks the Fermilab Theory Group for hospitality during the completion of this work.

\section{References}

[1] M. Battaglia et al., Proposed post-LEP benchmarks for supersymmetry, arXiv:hepph/0106204.

[2] J. Ellis, T. Falk, G. Ganis and K. A. Olive, Phys. Rev. D62 (2000) 075010 [arXiv:hep$\mathrm{ph} / 0004169]$.

[3] Joint LEP 2 Supersymmetry Working Group, http://lepsusy.web.cern.ch/lepsusy/Welcome.html.

[4] LEP Higgs Working Group for Higgs boson searches, OPAL Collaboration, ALEPH Collaboration, DELPHI Collaboration and L3 Collaboration, Search for the Standard Model Higgs Boson at LEP, ALEPH-2001-066, DELPHI-2001-113, CERN-L3-NOTE-2699, OPAL-PN-479, LHWG-NOTE-2001-03, CERN-EP/2001-055, arXiv:hep-ex/0107029; Searches for the neutral Higgs bosons of the MSSM: Preliminary combined results using LEP data collected at energies up to 209 GeV, LHWG-NOTE-2001-04, ALEPH-2001057, DELPHI-2001-114, L3-NOTE-2700, OPAL-TN-699, arXiv:hep-ex/0107030. 
[5] M.S. Alam et al., [CLEO Collaboration], Phys. Rev. Lett. 74 (1995) 2885 as updated in S. Ahmed et al., CLEO CONF 99-10; BELLE Collaboration, BELLE-CONF-0003, contribution to the 30th International conference on High-Energy Physics, Osaka, 2000. See also K. Abe et al., [Belle Collaboration], arXiv:hep-ex/0107065; L. Lista [BaBar Collaboration], arXiv:hep-ex/0110010.

[6] H. N. Brown et al., [Muon g-2 Collaboration], Phys. Rev. Lett. 86, 2227 (2001) [arXiv:hep-ex/0102017].

[7] J. L. Feng and T. Moroi, Phys. Rev. D 61, 095004 (2000) [arXiv:hep-ph/9907319];

J. L. Feng, K. T. Matchev and T. Moroi, Phys. Rev. Lett. 84, 2322 (2000) [arXiv:hepph/9908309]; Phys. Rev. D 61, 075005 (2000) [arXiv:hep-ph/9909334]; J. L. Feng and K. T. Matchev, Phys. Rev. D 63, 095003 (2001) [arXiv:hep-ph/0011356].

[8] J. L. Feng, K. T. Matchev and F. Wilczek, Phys. Lett. B 482, 388 (2000) [arXiv:hep$\mathrm{ph} / 0004043]$.

[9] J. Ellis, T. Falk and K. A. Olive, Phys. Lett. B444, 367 (1998) [arXiv:hep-ph/9810360]; J. Ellis, T. Falk, K. A. Olive and M. Srednicki, Astropart. Phys. 13 (2000) 181 [arXiv:hep-ph/9905481].

[10] M. E. Gómez, G. Lazarides and C. Pallis, Phys. Rev. D61, 123512 (2000) [arXiv:hepph/9907261] and Phys. Lett. B487, 313 (2000) [arXiv:hep-ph/0004028]; R. Arnowitt, B. Dutta and Y. Santoso, Nucl. Phys. B 606, 59 (2001) [arXiv:hep-ph/0102181].

[11] J. Ellis, T. Falk, G. Ganis, K. A. Olive and M. Srednicki, Phys. Lett. B 510 (2001) 236 [arXiv:hep-ph/0102098].

[12] We use version 7.51 of H. Baer, F. E. Paige, S. D. Protopopescu and X. Tata, ISAJET 7.48: A Monte Carlo event generator for $p p, \bar{p} p$, and $e^{+} e^{-}$reactions, arXiv:hepph/0001086 with a couple of minor modifications, which have already been implemented in the current version 7.58 .

[13] Information about this code is available from K. A. Olive: it contains important contributions from T. Falk, G. Ganis, J. McDonald, K. A. Olive and M. Srednicki.

[14] G. Jungman, M. Kamionkowski and K. Griest, Phys. Rept. 267, 195 (1996) [arXiv:hepph/9506380]; http://t8web.lanl.gov/people/jungman/neut-package.html. 
[15] J. L. Feng, K. T. Matchev and F. Wilczek, Phys. Rev. D 63, 045024 (2001) [arXiv:astro$\mathrm{ph} / 0008115]$.

[16] Joint LEP 2 Supersymmetry Working Group, Combined LEP Chargino Results, up to $208 \mathrm{GeV}$, http://lepsusy.web.cern.ch/lepsusy/www/inos_moriond01/charginos_pub.html.

[17] Joint LEP 2 Supersymmetry Working Group, Combined LEP Selectron/Smuon/Stau Results, 183-208 GeV, http://alephwww . cern.ch/ ganis/SUSYWG/SLEP/sleptons_2k01.html.

[18] S. Heinemeyer, W. Hollik and G. Weiglein, Comput. Phys. Commun. 124, 76 (2000) [arXiv:hep-ph/9812320].

[19] G. Degrassi, P. Gambino and G. F. Giudice, JHEP 0012, 009 (2000) [arXiv:hepph/0009337]; see also M. Carena, D. Garcia, U. Nierste and C. E. Wagner, Phys. Lett. B 499, 141 (2001) [arXiv:hep-ph/0010003].

[20] L. L. Everett, G. L. Kane, S. Rigolin and L. Wang, Phys. Rev. Lett. 86, 3484 (2001) [arXiv:hep-ph/0102145]; J. L. Feng and K. T. Matchev, Phys. Rev. Lett. 86, 3480 (2001) [arXiv:hep-ph/0102146]; E. A. Baltz and P. Gondolo, Phys. Rev. Lett. 86, 5004 (2001) [arXiv:hep-ph/0102147]; U. Chattopadhyay and P. Nath, Phys. Rev. Lett. 86, 5854 (2001) [arXiv:hep-ph/0102157]; S. Komine, T. Moroi and M. Yamaguchi, Phys. Lett. B506, 93 (2001) [arXiv:hep-ph/0102204]; S. P. Martin and J. D. Wells, Phys. Rev. D 64, 035003 (2001) [arXiv:hep-ph/0103067]; H. Baer, C. Balazs, J. Ferrandis and X. Tata, Phys. Rev. D 64, 035004 (2001) [arXiv:hep-ph/0103280]; R. Arnowitt, B. Dutta, B. Hu and Y. Santoso, Phys. Lett. B505 (2001) 177 [arXiv:hep-ph/0102344].

[21] J. Ellis, D. V. Nanopoulos and K. A. Olive, Phys. Lett. B 508 (2001) 65 [arXiv:hep$\mathrm{ph} / 0102331]$.

[22] T. Ibrahim and P. Nath, Phys. Rev. D 62, 015004 (2000) [arXiv:hep-ph/9908443].

[23] A. Czarnecki, B. Krause and W. J. Marciano, Phys. Rev. D52 (1995) 2619 [arXiv:hepph/9506256]; Phys. Rev. Lett. 76 (1996) 3267 [arXiv:hep-ph/9512369].

[24] J. Ellis, J.S. Hagelin, D.V. Nanopoulos, K.A. Olive and M. Srednicki, Nucl. Phys. B238 (1984) 453; see also H. Goldberg, Phys. Rev. Lett. 50 (1983) 1419. 
[25] T. Falk, A. Ferstl and K. A. Olive, Phys. Rev. D59 (1999) 055009 [Erratum-ibid. D 60 (1999) 119904] [arXiv:hep-ph/9806413].

[26] M. A. Shifman, A. I. Vainshtein and V. I. Zakharov, Phys. Lett. 78B, 443 (1978).

[27] J. Ellis, A. Ferstl and K. A. Olive, Phys. Lett. B481 (2000) 304 [arXiv:hep-ph/0001005].

[28] J. Ellis, A. Ferstl and K. A. Olive, Phys. Rev. D63, 065016 (2001) [arXiv:hep$\mathrm{ph} / 0007113]$.

[29] J. Gasser, H. Leutwyler, and M. E. Sainio, Phys. Lett. B253 (1991) 252; M. Knecht, PiN Newslett. 15, 108 (1999) [arXiv:hep-ph/9912443].

[30] M. G. Olsson, Phys. Lett. B482, 50 (2000) [arXiv:hep-ph/0001203]; M. M. Pavan, R. A. Arndt, I. I. Strakovsky and R. L. Workman, PiN Newslett. 15, 118 (1999) [arXiv:nucl-th/9912034].

[31] R. Arnowitt, B. Dutta and Y. Santoso, arXiv:hep-ph/0008336.

[32] G. K. Mallot, in Proc. of the 19th Intl. Symp. on Photon and Lepton Interactions at High Energy LP99 ed. J.A. Jaros and M.E. Peskin, Int. J. Mod. Phys. A 15S1, 521 (2000) [eConf C990809, 521 (2000)] [arXiv:hep-ex/9912040].

[33] M. Drees and M. Nojiri, Phys. Rev. D 48, 3483 (1993) [arXiv:hep-ph/9307208].

[34] A. B. Lahanas, D. V. Nanopoulos and V. C. Spanos, Phys. Lett. B 518 (2001) 94 [arXiv:hep-ph/0107151].

[35] CDMS Collaboration, R. W. Schnee et al., Phys. Rept. 307, 283 (1998).

[36] CRESST Collaboration, M. Bravin et al., Astropart. Phys. 12, 107 (1999) [arXiv:hepex/9904005].

[37] H. V. Klapdor-Kleingrothaus, arXiv:hep-ph/0104028.

[38] N. J. Spooner et al., Phys. Lett. B 473, 330 (2000).

[39] DAMA Collaboration, R. Bernabei et al., Phys. Lett. B 436, 379 (1998).

[40] J. Ellis, A. Ferstl and K. A. Olive, in preparation. 
[41] J. Silk, K. Olive and M. Srednicki, Phys. Rev. Lett. 55, 257 (1985); M. Srednicki, K. A. Olive and J. Silk, Nucl. Phys. B279, 804 (1987); T. K. Gaisser, G. Steigman and S. Tilav, Phys. Rev. D34, 2206 (1986); J. S. Hagelin, K. W. Ng and K. A. Olive, Phys. Lett. B180, 375 (1986); K. Ng, K. A. Olive and M. Srednicki, Phys. Lett. B188, 138 (1987).

[42] K. Freese, Phys. Lett. B167, 295 (1986); L. M. Krauss, M. Srednicki and F. Wilczek, Phys. Rev. D33, 2079 (1986); A. Gould, J. A. Frieman and K. Freese, Phys. Rev. D39, 1029 (1989).

[43] H. Baer and M. Brhlik, Phys. Rev. D 57, 567 (1998) [arXiv:hep-ph/9706509]; A. Corsetti and P. Nath, Int. J. Mod. Phys. A 15, 905 (2000) [arXiv:hep-ph/9904497]; A. B. Lahanas, D. V. Nanopoulos and V. C. Spanos, Phys. Rev. D 62 (2000) 023515 [arXiv:hepph/9909497]; A. Bottino, F. Donato, N. Fornengo and S. Scopel, Phys. Rev. D 63, 125003 (2001) [arXiv:hep-ph/0010203]; L. Roszkowski, R. Ruiz de Austri and T. Nihei, JHEP 0108, 024 (2001) [arXiv:hep-ph/0106334].

[44] V. Barger, F. Halzen, D. Hooper and C. Kao, arXiv:hep-ph/0105182.

[45] AMANDA Collaboration, C. Spiering et al., talk given at the 8th International Workshop on Neutrino Telescopes, Venice, Italy, 23-26 February 1999, arXiv:astroph/9906205.

[46] NESTOR Collaboration, L. K. Resvanis et al., talk given at the 8th International Workshop on Neutrino Telescopes, Venice, Italy, 23-26 February 1999.

[47] ANTARES Collaboration, J. R. Hubbard et al., HE.6.3.03 and ANTARES Collaboration, L. Moscoso et al., HE.6.3.04 in Proceedings of the 26th International Cosmic Ray Conference (ICRC 99), Salt Lake City, Utah, 17-25 August 1999.

[48] M. Leuthold, Prepared for International Workshop on Simulations and Analysis Methods for Large Neutrino Telescopes, Zeuthen, Germany, 6-9 Jul 1998.

[49] L. Bergström, J. Edsjö and M. Kamionkowski, Astropart. Phys. 7, 147 (1997) [arXiv:astro-ph/9702037]; L. Bergström, J. Edsjö and P. Gondolo, Phys. Rev. D58, 103519 (1998) [arXiv:hep-ph/9806293].

[50] T. Damour and L. M. Krauss, Phys. Rev. Lett. 81, 5726 (1998) [arXiv:astroph/9806165]; L. Bergström, T. Damour, J. Edsjö, L. M. Krauss and P. Ullio, JHEP 9908, 010 (1999) [arXiv:hep-ph/9905446]. 
[51] M. Urban, A. Bouquet, B. Degrange, P. Fleury, J. Kaplan, A. L. Melchior and E. Pare, Phys. Lett. B293, 149 (1992) [arXiv:hep-ph/9208255]; V. S. Berezinsky, A. V. Gurevich and K. P. Zybin, Phys. Lett. B294, 221 (1992); V. Berezinsky, A. Bottino and G. Mignola, Phys. Lett. B325, 136 (1994) [arXiv:hep-ph/9402215].

[52] L. Bergström, P. Ullio and J. H. Buckley, Astropart. Phys. 9, 137 (1998) [arXiv:astro$\mathrm{ph} / 9712318]$.

[53] GLAST Collaboration, A. Moiseev et al., HE.5.1.02 in Proceedings of the 26th International Cosmic Ray Conference (ICRC 99), Salt Lake City, Utah, 17-25 August 1999; H. F. Sadrozinski, Nucl. Instrum. Meth. A 466, 292 (2001).

[54] MAGIC Collaboration, M. Martinez et al., OG.4.3.08 in Proceedings of the 26th International Cosmic Ray Conference (ICRC 99), Salt Lake City, Utah, 17-25 August 1999.

[55] J. F. Navarro, C. S. Frenk and S. D. White, Astrophys. J. 462, 563 (1996) [arXiv:astro$\mathrm{ph} / 9508025]$.

[56] A. J. Tylka, Phys. Rev. Lett. 63, 840 (1989); M. S. Turner and F. Wilczek, Phys. Rev. D42, 1001 (1990); M. Kamionkowski and M. S. Turner, Phys. Rev. D43, 1774 (1991).

[57] I. V. Moskalenko and A. W. Strong, Phys. Rev. D60, 063003 (1999) [arXiv:astro$\mathrm{ph} / 9905283]$.

[58] AMS Collaboration, S. Ahlen et al., Nucl. Instrum. Meth. A 350, 351 (1994); A. Barrau [AMS Collaboration], arXiv:astro-ph/0103493.

[59] J. Silk and A. Stebbins, Astrophys. J. 411, 439 (1993). 